\title{
THE NONPOSITIVITY OF SOLUTIONS TO PSEUDOPARABOLIC EQUATIONS
}

\author{
WILLIAM RUNDELL AND MICHAEL STECHER
}

Abstract. Conditions are given on the nonnegative data $\phi(x)$ and $g(x, t)$ such that solutions of the pseudoparabolic inequality $P[u]=(L-I) u_{t}+$ $L u<0$ in $D x(0, \tau)$

$$
\begin{aligned}
& u(x, 0)=\phi(x), \quad x \in D, \\
& u(x, t)=g(x, t), \quad x \in D \times(0, \tau),
\end{aligned}
$$

satisfy $u(x, t)>0$ in $D \times(0, \tau)$. Here $D$ is an open set in $\mathbf{R}^{n}$ and $L$ is a second order elliptic differential operator. A counterexample is provided to show that this condition is in a sense necessary. The result implies that solutions $P[u]=0$ do not in general satisfy a maximum principle.

1. Introduction. In a recent paper [2] the positivity of solutions of the pseudoparabolic equation

$$
\begin{aligned}
P[u] & =(L-I) u_{t}+L u=f, \quad(x, t) \in D \times(0, T), \\
u(x, 0) & =\phi(x), \quad x \in D \\
u(x, t) & =g(x, t), \quad(x, t) \in \partial D \times(0, T),
\end{aligned}
$$

was claimed under the assumption that $u(x, t)$ is a strong solution of (1.1), that the initial and boundary data $\phi(x)$ and $g(x, t)$ respectively are nonnegative and $f(x, t)<0$. The purpose of this note is to point out a flaw in this theorem and to show that in general the result is not true. Although it is possible to show that the solution of (1.1) will be nonnegative provided restrictions are placed on the data. The fact that this positivity result is not true in general implies that solutions of $P[u]=0$ may not satisfy a maximum principle as was claimed in [2]. Indeed we construct a solution of

$$
\begin{aligned}
P[u] & =0, \quad(x, t) \in D \times(0, T), \\
u(x, 0) & =\phi(x), \quad x \in D, \\
u(x, t) & =g(x, t), \quad(x, t) \in D \times(0, T),
\end{aligned}
$$

such that $0<\phi(x), g(x, t)<C$, and where $u(x, t)>C$ for some $(x, t) \in$ $D \times(0, T)$.

Received by the editors October 10, 1977 and, in revised form, June 30, 1978.

AMS (MOS) subject classifications (1970). Primary 35B99, $35 \mathrm{Q} 99$. 
Throughout this note we will employ the following notation:

$C^{m+\alpha}(D)$ denotes the Banach space of real valued functions which are $m$-times differentiable and whose derivatives are Holder continuous with exponent $\alpha(0<\alpha<1)$, $C_{0}^{m+\alpha}(D)$ denotes the closure in the usual $C^{m+\alpha}(D)$ norm of those $C^{m+\alpha}(D)$ functions with support compactly contained in $D$.

We assume that $D$ is a bounded open subset of $\mathbf{R}^{n}$ whose boundary, $\partial D$, is of class $C^{2+\alpha}$;

$$
L u=\sum_{i, j=1}^{n} a_{i j}(x) \frac{\partial^{2} u}{\partial x_{i} \partial x_{j}}+\sum_{i=1}^{n} b_{i}(x) \frac{\partial u}{\partial x_{i}}-c(x),
$$

where $L$ is a uniformly elliptic operator in $D$ whose coefficients lie in $C^{\alpha}(\bar{D})$ and $c(x)>0$

$$
u(x, 0)=\phi(x) \in C^{2+\alpha}(\bar{D}), \quad x \in \bar{D}
$$

$g(x, \cdot)$ is continuously differentiable, and $g(\cdot, t) \in C^{2+\alpha}(\bar{D}), t \in[0, T]$, and

$$
u(x, t)=g(x, t), \quad(x, t) \in \partial D \times(0, T) .
$$

We note that the elliptic operator $L-I$ can be considered as a map from $C_{0}^{2+\alpha}(D) \rightarrow C^{\alpha}(\bar{D})$ and as is well known this mapping has a bounded inverse $(L-I)^{-1}$ which we will denote by $-A$.

2. A nonpositivity result. In [2] it is claimed that if the data in (1.5) and (1.6) are nonnegative and $f<0$, then the solution $u(x, t)$ of $(1.1)$ is also nonnegative. The proof of this result is broken into two parts. The first, which is correct, covers the case when the data $g(x, 0)=0=\phi(x)$ for $x \in \partial D$. The second part of the proof is an attempt to avoid the above restriction on the data. The argument employed approximates the given data by data which is zero at the corners in an $L^{2}$ norm and then it is claimed that the approximating solutions will converge to the desired solution. This is incorrect. To force the approximating solutions to converge the data must also converge but in a smoother norm than $L^{2}$, e.g. $W^{1,2}$. The convergence in this norm will imply pointwise convergence everywhere of the data and hence the restriction that the data be zero at the corners cannot be removed by this argument. In fact we show below that the solution to (1.1) may become negative. To facilitate our analysis we perform a preliminary calculation.

$$
\text { Put } u=e^{-t} v \text {. }
$$

Then we have

$$
\begin{aligned}
\hat{P}[v] & =(L-I) v_{t}+v=e^{t} P[u]=e^{t} f, \\
v(x, 0) & =\phi(x), \quad x \in D, \\
v(x, t) & =e^{t} g(x, t), \quad(x, t) \in \partial D \times(0, T) .
\end{aligned}
$$


Define $G(x, t)$ by

$$
\begin{aligned}
(L-I) G & =0, \quad(x, t) \in D \times[0, T), \\
G(x, t) & =e^{t} g(x, t), \quad(x, t) \in \partial D \times[0, T) .
\end{aligned}
$$

Since $g(x, t)>0$ we have by the maximum principle for elliptic equations [1] that $G(x, t) \geq 0$. We also note that $G(x, t) \in C^{2+\alpha}(\bar{D}) \cap C^{1}(0, T)$. Note that if $g(x, t)=\phi(x) \psi(t)$ with $\psi(0)=1$, then $G(x, t)=G(x, 0) e^{t} \psi(t)$. Define $w(x, t)$ by

$$
w=v-G .
$$

Then $w \in C_{0}^{2+\alpha}(\bar{D}) \cap C^{1}(0, T)$ and

$$
\begin{aligned}
\hat{P}[w] & =\hat{P}[v]-\hat{P}[G]=e^{t} f-G, \quad(x, t) \in D \times(0, T), \\
w(x, 0) & =\phi(x)-G(x, 0), \quad x \in D, \\
w(x, t) & =0, \quad(x, t) \in \partial D \times(0, T) .
\end{aligned}
$$

We now rewrite (2.5) as a differential equation in the space $C^{\alpha}(\bar{D})$

$$
\begin{aligned}
w_{t}+(L-I)^{-1} w & =(L-I)^{-1}\left\{e^{t} f-G\right\}, \\
w(x, 0) & =\phi(x)-G(x, 0) .
\end{aligned}
$$

Integrating (2.6) from 0 to $t$ we have

$$
\begin{aligned}
w(t) & =\int_{0}^{t} A w(\tau) d \tau+h(t), \text { and } \\
h(t) & =\int_{0}^{t} A\left\{G-e^{t} f\right\} d \tau+\phi(x)-G(x, 0),
\end{aligned}
$$

where $A$ denotes the bounded linear operator $-(L-I)^{-1}$ which maps $C^{\alpha}(\bar{D})$ into $C_{0}^{\alpha}(\bar{D})$. We note again that from the maximum principle for elliptic operators it follows that $u>0$ implies $A u>0$. Observe that the integrand in (2.7b) is nonnegative. Thus if we assume

$$
\phi(x)-G(x, 0) \geq 0,
$$

then it is easy to see by Picard interation that $w$ and hence $v$ and $u$ are nonnegative on $D \times(0, T)$. We remark that if $u(x, t)$ is zero for $(x, t) \in \partial D$ $\times\{0\}$, then (2.8) follows. Picard iteration also shows that if $h<0$ on $D \times(0, T)$ then the solution $w$ of $(2.7)$ also satisfies $w<0$. We summarize as follows:

THEOREM. Let $u(x, t)$ be the solution to (1.1) where $f(x, t)<0$ and the data $g(x, t)$ and $\phi(x)$ are nonnegative. If in addition (2.8) holds, then $u(x, t)>0$.

We now show that it is possible to pick $g(x, t), \phi(x)$, and $f(x, t)$ so that $h(x, t)<0, h \neq 0$.

LemMa 1. Let $c$ be any positive constant. Define $G(x, 0)$ by

$$
(L-I) G(x, 0)=0, \quad G(x, 0)=c, x \in \partial D .
$$

Then there is a $\delta>0$ such that

$$
0<\delta<G(x, 0)<c .
$$


Proof. The upper bound follows from the maximum principle; the lower bound follows from the fact that $G$ attains a positive minimum.

Since $G>0$ the maximum principle for elliptic equations shows again that $A G(x, 0)>0$. Thus from Lemma 1 we can find $\varepsilon>0$ so that

$$
0<G(x, 0)-\varepsilon A G(x, 0)<c .
$$

Since the gradient of $G(x, 0)-\varepsilon A G(x, 0)$ is bounded in $\bar{D}$ we may pick $\phi(x) \in C^{2+\alpha}(\bar{D})$ so that

$$
0 \leqslant \phi(x) \leqslant G(x, 0)-\varepsilon A G(x, 0), \quad \phi(x)=c, x \in \partial D .
$$

The $\phi(x)$ of (2.11) will be used as the initial data in our counterexample.

LEMMA 2. Let $\psi(t)$ be any continuously differentiable function of $t$ such that

$$
0<\psi(t)<1, \quad \psi(0)=1, \quad \operatorname{supp} \psi \subset[0,1], \text { and } \int_{0}^{1} \psi(t) d t<\varepsilon / e,(2.13)
$$

where $\varepsilon$ is as in (2.11). Let $g(x, t)=\phi(x) \psi(t)$. Let $h(x, t)$ be defined by (2.7b), where we set $f(x, t)=0$. Then $h(x, t)<0$ in $D \times[0, T)$.

ProOF.

$$
\begin{aligned}
h(x, t) & =\int_{0}^{t} A G(x, \tau) d \tau+\phi(x)-G(x, 0) \\
& =\int_{0}^{t} e^{\tau} \psi(\tau) A G(x, 0) d \tau+\phi(x)-G(x, 0) \\
& =A G(x, 0) \int_{0}^{t} e^{\tau} \psi(\tau) d \tau+\phi(x)-G(x, 0) \\
& <\varepsilon A G(x, 0)+\phi(x)-G(x, 0)<0
\end{aligned}
$$

by (2.3), (2.12), and (2.11).

For this $h$ the solution $w$ of $(2.7 \mathrm{a})$ is $<0$. Since $v=w+G$ and $G(x, t)=$ 0 for $t>1$ we have that $v(x, t)<0$ for $t>1$. Thus $u(x, t)=e^{t} v(x, t)$ and the solution of (1.1) also takes on negative values.

To see that the maximum principle will not hold in general take $L$ with $c(x) \equiv 0$. Let $u_{1}$ be the above constructed solution with this $L$. Set $u=c-$ $u_{1}$. Then

$$
\begin{aligned}
P[u] & =0, \quad(x, t) \in D \times(0, T), \\
0 & <c-\phi(x)=u(x, 0)<c, \quad x \in D \\
0 & <c-\psi(t) \phi(x)=u(x, t)<c, \quad(x, t) \in \partial D \times(0, T) .
\end{aligned}
$$

Since $u_{1}(x, t)$ becomes negative we have $u(x, t)>c$ for $t>1$.

\section{BIBLIOGRAPHY}

1. M. H. Protter and M. F. Weinberger, Maximum principles in differential equations, PrenticeHall, Englewood Cliffs, N. J., 1976.

2. M. Stecher and W Rundell, Maximum principles for pseudoparabolic partial differential equations, J. Math. Anal. Appl. 57 (1977), 110-118.

Department of Mathematics, Texas A \& M University, College Station, Texas 77843 